Jean Yves Astier ${ }^{1}$, Igor Yurievich Zhukov², Oleg Nikolaevich Murashov ${ }^{2}$

${ }^{1}$ HyperPanel Lab, Saclay 4 Rue Rene Razel Building'azur 91400 Saclay, France

e-mail:jean-yves.astier@hyperpanel.fr,.ORCID 0000-0002-7002-1195

${ }^{2}$ CEO of Ltd. «The National Mobile Portal»,

Volgogradskiy pr.,2 off.36, Moscow, 109316, Russia

e-mail:i.zhukov@inbox.ru,ORCID0000-0002-4429-8799; e-mail:olegxozbox@yandex.ru, ORCID 0000-0002-4467-2170

\title{
Smart Building Management Systems and Internet of Things
}

DOI: http://dx.doi.org/10.26583/bit.2017.3.02

Abstract. After a brief historical reminder on SCADA systems, we will present the new challenges regarding modern building technical management (BTM) systems. We identify the technological evolutions, which allow us to address these new issues, and describe the software and hardware architectures of our Building Management Internet of Things (BMIoT) solution. We end by comparing with other older solutions and by a short description of the new business models, our solution allows.

Keywords: Smart Building, Management Systems, Internet of Things (IoT)

For citation. ASTIER, Jean Yves; ZHUKOV, Igor Yurievich; MURASHOV, Oleg Nikolaevich. Smart Building Management Systems and Internet of Things. IT Security, [S.1.], v. 24, n. 3, p. 18-29, july 2017. ISSN 2074-7136. Available at: <https://bit.mephi.ru/index.php/bit/article/view/260>. Date accessed: $01 \mathrm{dec} .2017$. doi:http://dx.doi.org/10.26583/bit.2017.3.02.

Жан Ив Астье ${ }^{1}$, Игорь Юрьевич Жуков ${ }^{2,}$ Олег Николаевич Мурашов ${ }^{2}$

${ }^{1}$ HyperPanel Lab, Saclay 4 Rue Rene Razel Building'azur 91400 Saclay, France

e-mail:jean-yves.astier@hyperpanel.fr,.ORCID 0000-0002-7002-1195

${ }^{2}$ ООО «Национальньй мобильный портал»,

Волгоградский пр., 2, офис 36, Москва, 109316, Россия

e-mail:i.zhukov@inbox.ru, ORCID 0000-0002-4429-8799;

e-mail:olegxozbox@yandex.ru,ORCID0000-0002-4467-2170

\section{СИСТЕМЫ УПРАВЛЕНИЯ «УМНЫЙ ДОМ» И ИНТЕРНЕТ ВЕЩЕЙ \\ DOI: http://dx.doi.org/10.26583/bit.2017.3.02}

Аннотащия. После краткой исторической справки о системах SCADA, мы дадим представление о новых задачах, связанных с современными подходами к построению систем технического управления. Далее мы остановимся на технологических достижениях, которые позволят нам решать эти новые задачи, и дадим описание программно-аппаратной архитектуры построения управляющей системы Интернета вещей. В заключение мы приведем сравнение с другими более традиционными решениями и дадим краткое описание новых бизнес-моделей, которые позволят реализовать наше новое решение поставленных задач.

Ключевые слова: Smart Building, Management Systems, Internet of Things (IoT)

Для иитирования. АСТЬЕ, Жан Ив; ЖУКОВ, Игорь Юрьевич; МУРАШОВ, Олег Николаевич. СИСТЕМЫ УПРАВЛЕНИЯ «УМНЫЙ ДОМ» И ИНТЕРНЕТ ВЕЩЕЙ. Безопасность информационнЫХ технологий, [S.1.], v. 24, n. 3, p. 18-29, july 2017. ISSN 2074-7136. Доступно на: $<$ https://bit.mephi.ru/index.php/bit/article/view/260>. Дата доступа: $01 \quad \mathrm{dec} .2017$. doi:http://dx.doi.org/10.26583/bit.2017.3.02.

\section{A Bit of History}

The building technical management systems[1-5] have appeared in the 60s, as one possible application of the Supervisory Control And Data Acquisition (SCADA) systems. The first 
systems are typically designed around a single central computer, which is connected by RS-485 serial cables to electronic blocs called Remote Terminal Units (RTUs). (Figure 1).

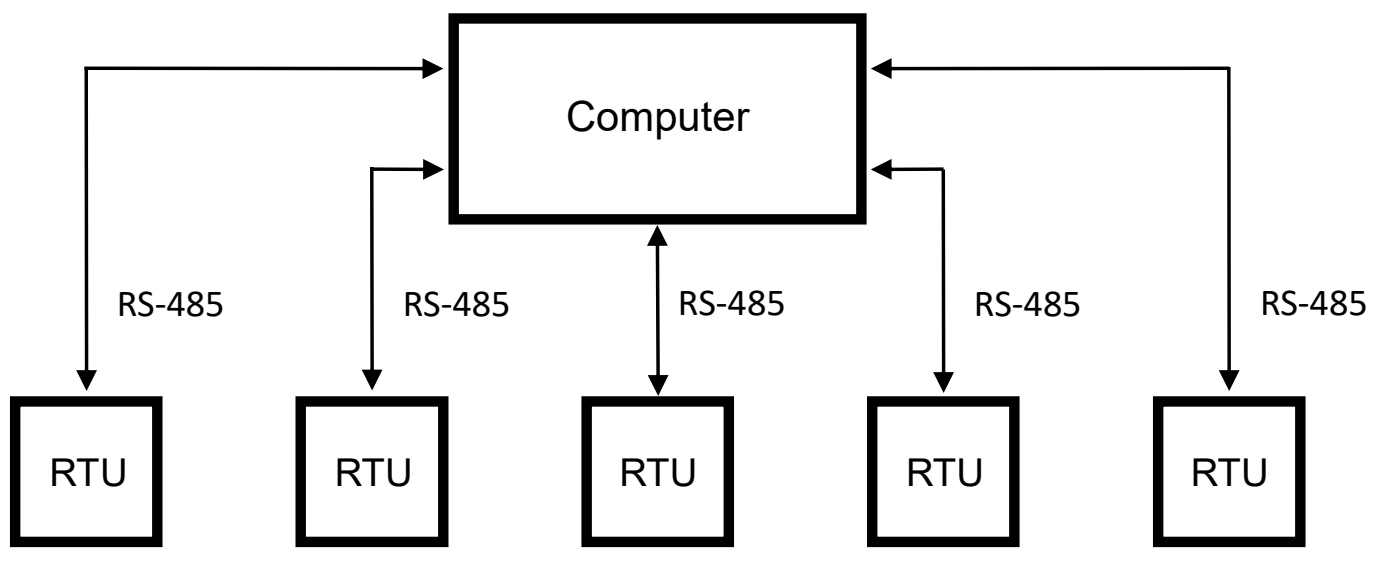

Figure 1: The first systems are typically designed around a single central computer

The RTUs don't perform any processing of the acquired data. They can only perform two types of commands: reads and writes. The protocols used between the central computer and the remote terminals are often proprietary, which makes it impossible to share the serial cable with other applications. The central computer periodically polls all of the measured values; the remote terminals do not detect the change in values.

\section{New Challenges}

\section{Considerable increase in the number of points}

In the $60 \mathrm{~s}$, a single all or nothing value was used to control the lighting in an entire floor or an entire zone, and there was no presence sensor. In modern buildings, each striplight is individually remote controlled, according to the information provided by the numerous presence sensors. For the same building surface area, the number of required data point for the lighting alone has increased 50- or 100-fold.

Since the 90s, two majors Wide-Area Networks (WAN) [6,7] have appeared and have been massively deployed: GSM and the Internet. As a result, a single BTM system is required to manage building separated by kilometers or more.

To give an approximate and arbitrary order or magnitude, the number of points of a considerable BTM system has gone from a few thousand to a few hundred thousand.

\section{New Data Types}

For decades, the SCADA systems - building management systems included - only had to handle all or nothing (AON) values, analog measurement or target values of up to 32 bits (ANA), or small character strings (STR).

The digitization of images and their compression (JPEG) on one hand, and the use of standard protocols (Ethernet) for the RTUs on the other have made possible the interconnect of image capture devices, fixed or animated, on the local network used by the BTM. Request to integrate these images into the BTM systems followed. In addition to these input images, a BTM can also generate output images, typically for screens which will replace older character-based displays.

\section{Geographic Point Repartition}

As previously mentioned, there are requests for multi-site BTM systems, which have two major consequences: 
- This requires that the central system communicate with the RTUs through WANs, using therefore standard protocols (IP).

- But also more importantly, constantly polling all the points becomes wholly unfeasible.

\section{Cloud and the Internet}

As with telecommunications hardware, it is increasingly expected that building management systems can be remotely managed. On one hand the RTUs can be far from the central system, but also the terminals of the personnel in charge can be far from the central system. To be more precise, it is requested that there be no difference in the management terminals whether they be close or far from the central computer. Multi-screen personnel computers, tablets and smartphones must all be able to be used; it is accepted that a terminal with less screen real estate cannot display as large overview displays, or risk becoming too small to be readable.

\section{Increase in Data Model Complexity}

As long as the number of points in the BTM system was limited (a few thousand), and that the responsibility of the supervised points was left to a single entity, a strictly hierarchical organization of the points was entirely sufficient. Each point belongs to a single zone, each zone belongs to a single floor, and each floor belongs to a single building. A natural representation of such an organization is that of folders, as shown in any computer file explorer.

When both the number of points increases (hundreds of thousands), and the responsibility of these points is split across independent and sometimes competing organizational entities, a hierarchical organization is no longer suitable. At a very fundamental level, it is necessary to be able to use filtering and joining operations, which from a computer science point of view are much more complex to model than a simple up down navigation as used in a hierarchical model. The data organization models called "relational" have two base operations, which are filtering and joining, which are made available through dedicated data manipulation languages such as the Structured Query Language (SQL).

\section{Dramatic Reduction in Costs}

The multiplication of the number of supervised points in the buildings automatically drives demands for lower unit cost for the points. We mean by that the Non-Recurring Expenses (NREs) must be lowered, but also that the recurring expenses (operational costs) must also be lowered.

Technical progress automatically reduces the costs hardware, in particular that of the Remote Terminal Units (RTUs) which furthermore have become capable of performing certain automation functions by programming, and become Programmable Logic Controllers (PLCs).

With regards to human costs, in particular those of the cable laying personnel and the home automation specialist who configures all the points, there are two parameters on which we can act: reducing the necessary time, and reducing the hourly rates by simplifying the tasks to perform. The work methodology used for BTM systems comprising a few thousand points can't be extended when that number reaches the few hundred thousand.

\section{Sustainability}

The first BTM systems comprised specific elements, both for the hardware (RTUs) and for the software. In particular the software was usually created on a case-by-case basis by services companies, and the project teams were sometimes dissolved even before the BTM was put into production, due to delays.

All of this has vastly evolved in part because of the standardization of the SCADA protocols[8], making equipment interchangeable, and in part because of SCADA and BTM software whose sustainability is largely greater than that of specific software development. 
Nonetheless, today's BTM systems still suffer from major lack of sustainability:

- Configuration files, which contain the data describing the points and their organization, sometimes use a proprietary format, and sometimes are not even stored on the machines that comprise the BTM system.

- The measured data, the value of the points is sometimes also proprietary.

- Many existing professional software require specific know-how, which is to say personal specifically trained to use it. Moreover, even if the software itself is maintained, a reduction in the number of new deployments leads to a reduction and eventually disappearance of the required experience in the work force.

\section{Life Cycle}

The considerable increase in the number of supervised points in each building means that every day new points appear and other disappear. Faced with a daily flow of incoming and outgoing points, it is no longer possible to completely stop the operations of a BTM system to perform these additions and removals.

More generally, very large deployments covering multiple geographic sites face new "life cycle" challenges. A modern BTM system used in large configurations must be able to do the following:

- During the initial deployment, allow immediate verification of the cabling and a precise diagnostic in case of error, in order to limit the comings and goings of the cable layout personnel. In particular, this concerns the command of the lighting in the dropped ceiling. This entails that the initial description be available before the cabling even starts, and that it be possible to "manually" activate each of the points.

- Dynamically modify the configuration data. As explained above, the solutions based on configuration files which must be compiled before being used, sometimes a minutes long operation, are no longer viable.

\section{Security}

Almost all security[9] vulnerabilities impact and are caused by some very widely spread technical building blocks clearly identified:

- Windows Server

- All non-validated Linux versions

- HTML/JavaScript Frameworks

So avoiding the use of those is a mandatory point.

\section{Going from "cost center" to "profit center"}

The final and most important change is the change in business model which BTM systems take part in. Contrary to SCADA systems used for instance in production lines, where the end goal is to improve productivity, that is an economic target, the first BTM were more of prestige spending, where the maintenance costs of the hardware (computer and RTUs) was more than the energy economies (heating and lighting) it allowed.

A modern BTM system on the other hand is part of an overall economic objective. It must first of all lead to significant energy savings on lighting and heating. Also, when individual homes are being supervised, the infrastructure can be used for other purposes and not only for the technical management of the building (heating, lighting, and energy). We go from a BTM system to an IoT management system, where some IoT devices are BTM sensors and others are not, individual medical sensors for instance.

Like business management computer systems, which were gradually outsourced from companies to become an economic activity in its own right, a similar evolution can be expected concerning BTM or IoT services, which unavoidable bottom line constraints. 


\section{Major Technological Changes}

\section{Replacement of Vector Data by Bitmap Data}

Up to the $80 \mathrm{~s}$, there were two types of user terminals: the "alphanumeric" terminals, and the "graphical" terminals, using random scan mode only. The second were uncommon due to their high price. In the case of SCADA systems, a third type of display time was often used; custom-built overview displays on control room walls using lamps and electromechanical displays driven by the central computer using a custom-built RTU.

The 90s will see wide-spread use of graphical card using "bitmap" memory, which will replace both the "alphanumeric" and "graphical" screens. However the BTM continue to use vector graphics because CAD software used by architects produces vector graphics. As we will see later, there is no longer any reason to use vector graphics in BTM software.

\section{Changes in Professional Software}

All wide-spread graphical software tools are able to produce files in Web bitmap format, JPEG or PDF. This is true in particular of software used by the workers of the construction and equipment production fields, that is to say software used by architects, sensor providers, and RTU or PLC providers. In the case of BTM systems, millimeter level precision of a 3D vector format such as the DXF format is useless, a nice artist's rendering or a bitmap plan are preferred when the human interface of the BTM is using web technologies.

Given the definition of the points, the XLS format is ideal. In particular, all spreadsheet software can product CSV format files, which all database software can then import, for instance using PHP scripts.

\section{Changes in Graphics Software}

The two leading pieces of software from the Adobe corporations, Photoshop and Illustrator, are, in terms of software development costs, colossal. It is entirely impossible to produce as many features and as much power when a SCADA or BTM piece of software, when the developer of the overview is only a small part of the overall cost of the product.

Moreover, these very powerful programs have a readily available large amount of trained users. Finally the web provides a nearly unlimited library of graphics. To create simple animations for the overviews, the creation of an animated GIF file is much cheaper than that of a script for a SCADA or BTM application.

All wide-spread graphical software tools are able to produce files in Web bitmap format, JPEG or PDF. This is true in particular of software used by the workers of the construction and equipment production fields, that is to say software used by architects, sensor providers, and RTU or PLC providers. In the case of BTM systems, millimeter level precision of a 3D vector format such as the DXF format is useless, a nice artist's rendering or a bitmap plan are preferred when the human interface of the BTM is using web technologies.

Given the definition of the points, the XLS format is ideal. In particular, all spreadsheet software can product CSV format files, which all database software can then import, for instance using PHP scripts.

\section{Changes in DBMS}

The relational data model is considered to have appeared in 1970, the year when Edgar Frank Codd published a seminal article entitled A Relational Model of Data for Large Shared Data Banks. In 1979, the Relation Software Inc. company (now Oracle Corporation) begins the commercialization of the first version of SQL. In 1987, ISO published the international standard ISO/CEI 9075 - Information technologies - Database languages - SQL. 
During 15 years or so, the costs of relational DBMS were very steep. Commercial practices of forced sales of expensive intellectual services (training and support) were common. Finally, as hardware support for the DMBS, it was necessary to acquire and maintain powerful and costly computers. For all these financial reasons, the editors of BTM programs were forced to keep their strictly proprietary point management systems, although the technical advantages of a relational model were increasingly obvious.

All of this has drastically changed since. First, there are free DBMS programs, which when correctly applied - can manage millions of points. Second, the cost of a computer able to support such a DBMS is around a thousand Euros, which is trivial when compared to the costs of cabling a single building.

\section{Changes in the Controllers}

Since the $80 \mathrm{~s}$, the significant reduction in processor and RAM memory costs have led to both a lowering of the costs of the RTUs, but also to an increase in their capabilities. From simple telecommunications hub, they have become genuine computers, that is to say with storage, data processing, and broad communications capabilities. Moreover, the protocols used have become standard, such as MODBUS/TCP.

Modern controllers, in addition to their processing capabilities, can handle so-called "fieldbus" protocols, while talking to the central system through a single unified protocol (MODBUS/TCP). The server software architecture is simplified, and the communication between the controllers and the server can occur through a WAN (Internet). Moreover the controllers are able to directly feed DBMS (remote SQL client).

\section{Consequences of these Changes in BTM}

It doesn't seem necessary to lead the reader and detail point by point the obvious advantages of the technical changes with regards to the previously outlined new challenges. We limit ourselves to underline the follow-on fact: many professional SCADA and BTM software has become obsolete:

- The functionality at the heart of these professional applications can be better provided by widely available cheap or free programs.

- Conversely, major issues such as the data exchange with the cloud, and security policy aspects are not at all taken into account.

\section{Introducing Our Solution}

\section{Hardware architecture}

This hardware architecture for a BMIoT system (Figure 2) is the one we have deployed on a site called Solaris in Clamart, France (www.solaris-energie-positive.com).

Specifications:

$30,000 \mathrm{~m}^{2}$ surface area;

116 geothermal probes $100 \mathrm{~m}$ under-ground;

$126 \mathrm{~km}$ of heating coil in the floor;

A $1,000 \mathrm{~m}^{2}$ in-door garden;

5,000 lights paired to a high-performance BTMS;

A new generation BTMS;

10 levels - 100 controllers - 24,000 controller points;

1,700 presence sensors - 450 energy meters;

1,000 real-time alarms;

6 heat pumps -11 central compressed air supplies. 


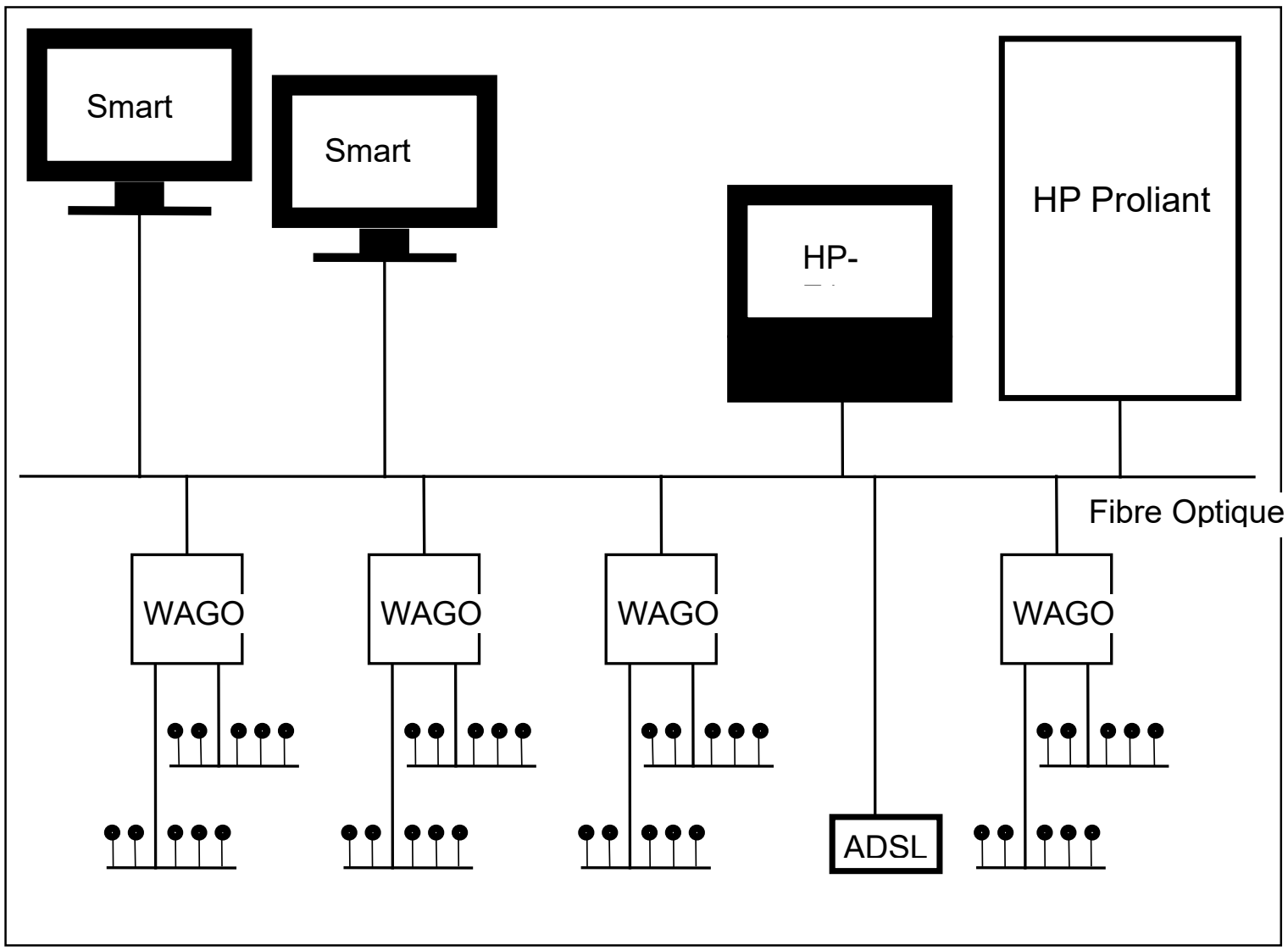

Figure 2: The hardware architecture for a BMIoT system

On the optical fiber are 100 WAGO controllers, an HP Proliant server, two HP-Z1 PCs (operator workstations), $50 \mathrm{TV}$ screens, and an ADSL Internet access.

\section{Software Architecture}

The fixed workstations are two Windows HP-Z1 PCs; the only software they need is a Web browser. The BTM server runs CentOS; it rust the Apache Web Server, PHP, and the MySQL relational DBMS. The remote workstations are Windows computers, tablets, or smartphones, the only required software being a web browser.

The WAGO controllers use the MODBUS/TCP protocol as well as a MySQL client which lets them directly use the MySQL DBMS. The software for the controllers is written using an automation language by the WAGO company.

The application layer of the BTM comprises two distinct applications. An administrative application is used to configure the BTM: number of floors, on zone, description of the 24,000 points. This description is stored in the DBMS; it can be used simultaneously by the administrative application, but also by the second application which is the supervision workstation. These two applications, these human interfaces are stored on the Proliant server, and are made of HTLM/JavaScript pages, which can be dynamically generated by PHP scripts on the server.

The display application used by the $50 \mathrm{TVs}$ is also written as a set of HTML/JavaScript pages. The displayed pages are periodically requested the TVs as HTTP GET requests. Some values displayed in the pages are themselves periodically obtained by the server from external web servers, such as road traffic conditions, or weather data.

One should note that no "framework" has been used for this web development for performance and security reasons. 
Overall Working (Figure 3)

1) All of the state changes which must raise an alert are all detected on the controllers which use their SQL client to directly perform a write in the DBMS (remote SQL UPDATE).

2) The values of the energy meters are stored in the DBMS every 10 minutes. The server performs the MODBUS/TCP read requests to the controllers, one after the other, which requires around 30 seconds for around 3,000 measurement points.

3) When a user workstation displays a zone map, the HTML/JavaScript human interface performs periodic polling to the WAGO controllers shown on the map, typically around 200 values. The human interface performs the read requests by MODBUS/TCP to the controllers in charge of the displayed points.

4) When a user workstation performs a command request, the human interface performs a MODBUS/TCP write directly to the relevant WAGO controller. The human interface also performs DBMS request which logs the request in order to maintain a history of the commands which were run.

5) Periodically, each connected TV runs an HTTP GET request on the server which dynamically generates a new HTML page (PHP script).

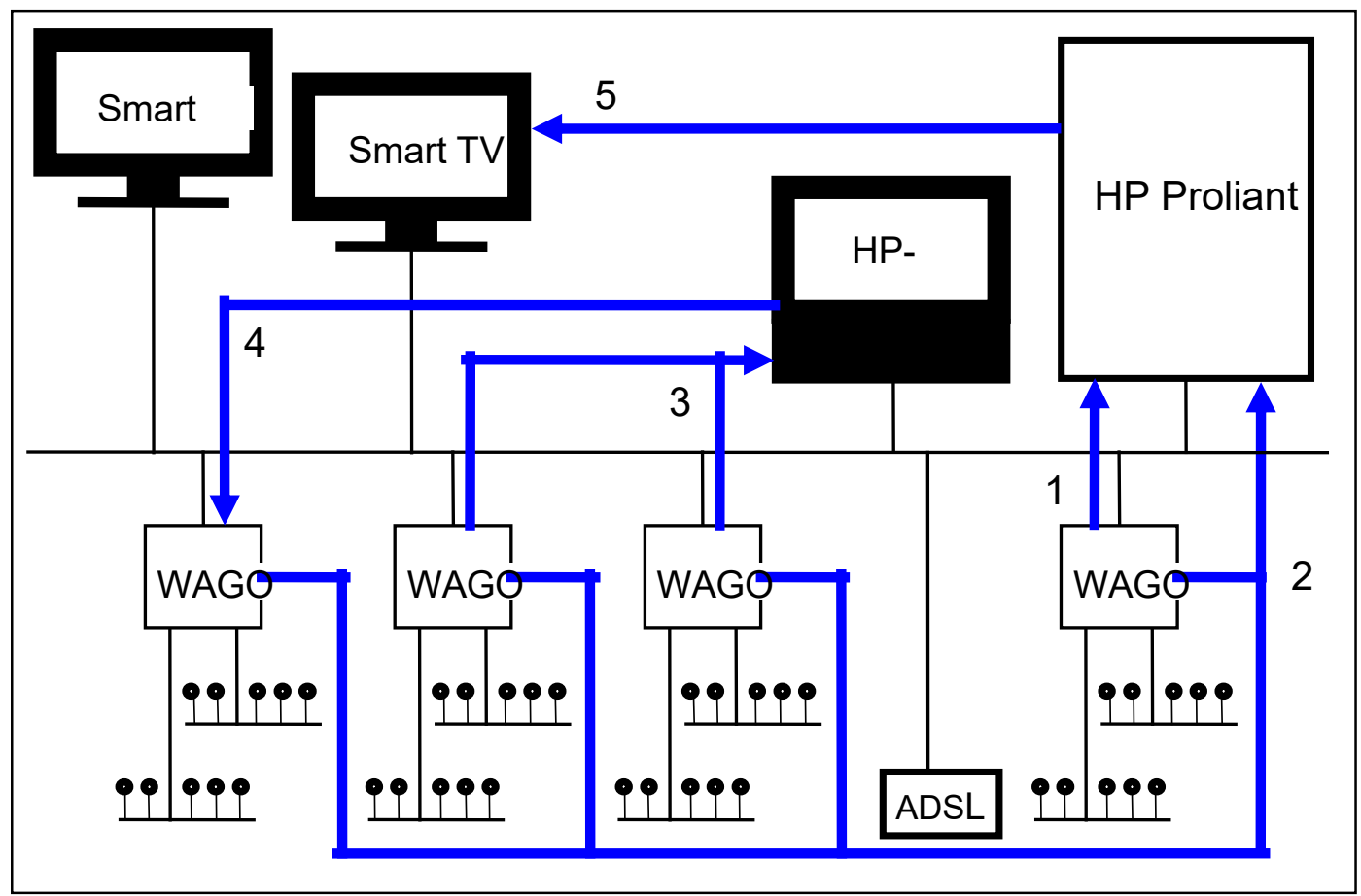

Figure 3: Overall Working

1 The alerts are directly sent by the controllers using remote SQL/TCP

2 The values of the meters are polled using MODBUS/TCP

3 A user workstation polls the values for the displayed zone using MODBUS/TCP

4 A user workstation performs a "manual" command using MODBUS/TCP

5 A screen read the values to display using an HTTP GET request

When software updates must be performed on the WAGO controllers, this is done using a Windows PC dedicated to this task and running an application provided by WAGO. This workstation is normally in the same room as the two fixed HP-Z1 stations. 
Operating this way minimizes the telecommunication transfers, which allows for remote workstations and permits the BTM server to be placed anywhere. Indeed, the 24,000 points are never all polled, contrary to other solutions. The remote workstations are secured using encryption (HTTPS or a VPN).

\section{Deployment and Installation Methodology}

Technically the use of a DBMS offers unrivaled operational flexibility. It also affords a more precise "life cycle" of every data point, in particular thanks to "empty values". The description information of every point can be provided progressively, and it is in no way required that the points of the same type be similarly filled. The definition of each point can be realized in 4 steps:

1) Identification of the points.

2) Placement of the points in an understandable space.

3) Detection of their "appearance".

4) Validation of proper function and expected behavior.

The identification of the points consists in giving a symbolic name to the point, a comment describing it (for example "Switch $26,4^{\text {th }}$ floor, zone 20"), and a code which gives its general type such as "switch", "light", "heat pump" without going into further detail. It is usually the building architect which provides this first level of point definition. This information is transferred, typically as an Excel export, to the personnel in charge of programming the controllers, so that the names of the points match.

The placement of the points is on a preview picture. Note that the preview image can be a scanned architect's plan, but that is not necessarily the case. Some images can be overviews drawn by a heating engineer. Others can be "artist's renderings" lacking the precision of an architect's plan, but which can be in the end easier to read. In the usual case, multiple representations will be used. The placement of a point is simply placing it on an image whose nature matters little. The data sources for this work are multiple. For instance the placement of the lighting and the light switches can be defined by the architect according to the demands of the client. The points having to do with the heating are defined by the heating engineer, and so on...

The detection of their physical "appearance". Both the installation of the controllers in the buildings as well as the cabling of the equipment (lighting, presence sensors, meters ...) occurs progressively, according to the availability of the teams of each of the trades. When tens of thousands or more are initially installed, it is a fantasy to believe that it is possible to wait for the end of the installation until the checks are performed. For instance, it is not efficient to discover issues in the ceiling lights after the drop ceiling tiles are installed. If the controllers for the lights are operational before the light installations are performed, the cabling can be immediately checked with the installing personnel still on site.

Validation of proper function and expected behavior. In addition to checking that the new equipments communicated properly, it is sometimes necessary in the case of more complex equipment (heating or cooling) to wait for more qualified personnel to perform further tests, which go beyond communications tests.

\section{Comparison}

We present bellow a quick comparison (Figure 4) of our solution and other profession software among the most complete and widespread SCADA, which are used to create BTM systems, that is to say the PCVUE32 program from the French company ARC Informatique. 


\begin{tabular}{|c|c|c|}
\hline SGBD & Central & Peripheral (archiving) \\
\hline Server OS & CentOS & Windows \\
\hline Graphics & Web bitmaps & Proprietary vector \\
\hline Maps & Web bitmaps & \\
\hline Programming languages & PHP, JavaScript & Basic 32 \\
\hline Telecoms & Only useful transfers & $\begin{array}{l}\text { Many useless transfers } \\
\text { (polling of the points) }\end{array}$ \\
\hline Data model & $\begin{array}{l}\text { Standard relational, which } \\
\text { allows an "organization" of } \\
\text { the data. }\end{array}$ & $\begin{array}{l}\text { Proprietary hierarchical, } \\
\text { which only allows "storing" } \\
\text { the data. }\end{array}$ \\
\hline Animations & GIF files (Web) & Basic 32 Programming \\
\hline Periodic actions & $\begin{array}{ll}\begin{array}{l}\text { Totally } \\
\text { (controllers) }\end{array} & \text { decentralized } \\
\end{array}$ & Centralized \\
\hline Development tools & All standard (web) & All custom \\
\hline Required personnel & Web developers & Specialized developers \\
\hline File formats & All standard (Web) & $\begin{array}{l}\text { All specific (overview, } \\
\text { symbols, variable and user } \\
\text { declaration). No DBMS. }\end{array}$ \\
\hline Remote stations & Same as local stations & Specific development \\
\hline License costs & $\begin{array}{l}\text { Flat-rate, independent of both } \\
\text { the number of points and } \\
\text { work stations. The number of } \\
\text { points need not predefined. }\end{array}$ & $\begin{array}{l}\text { Proportional to the number of } \\
\text { points and proportional to the } \\
\text { number of stations, which } \\
\text { leads to very steep costs with } \\
\text { just a few tens of thousands } \\
\text { of points. The number of } \\
\text { points must be known before- } \\
\text { hand. }\end{array}$ \\
\hline
\end{tabular}

Figure 4: Quick comparison of our solution and other profession software

One should note that there exist software products such as the ETS program from KNX which are not properly supervision applications. The ETS software is used to configure the parameters of the fieldbus (KNS home automation standard) statically. There are telecommunications devices on the market whose role is to convert the KNX protocol into standard protocols such as MODBUS/TCP for example, and let KNX hardware interconnect with our software solution, just as the controllers perform the conversion between fieldbuses and IP protocols.

\section{Business Model for our Solution}

The software solution which we have developed provides uniform supervision of both "typical" building equipment such as heating, lighting and energy consumption, as well as enduser IoT devices with new use-cases such as media consumption meters or health-related devices.

Its goal is to facilitate the transition of companies for maintenance in good operation conditions of buildings or facility management to a business model of Internet Player[10]. The main point of this transition lies in going from "small data" of traditional BTM to the "big data" which is the foundation of new and additional economic activities.

A "big data" can be described by three major characteristics:

- A huge amount of data, 
- The sources of the data are both human, but also machines (IoT). In the latter case, the data is automatically uploaded to the Cloud for this Big Data.

- The data are no longer isolated individual values, such as a temperature or a meter value. It is aggregated contextual data: a place, a temperature, weather conditions at this point in time.

Once this "big data" is available and set up, it becomes possible to provide new services to both the managers of the buildings, and the inhabitants on the other. These new services will be built using "big data" technologies, especially "deep learning", artificial intelligence or automatic analysis of large volumes of data. This is how companies such as Google, Facebook, or Twitter operate with end users. There exist multiple similar "business" opportunities in the professional world.

We can see in chronological order three types of new services:

- Those having to do with the improvement and rationalization of the building management. "Big Data" can improve the preventative maintenance strategies, reducing the repair operations.

- Those having to do with the inhabitants of the buildings. "Big data" will go through heterogeneous data sets, such as the actions on lighting, on the TV and the various IoT devices, including the medical devices, and will detect very reliably suspicious status. The sole correlation between electric consumption curves of one day and the next one enables for detection of abnormal situations. Thus this "big data" is not limited to the management of a building and can start generating revenue.

Finally, with all this information, it might become possible to create a marketing profile of the inhabitants, which can be sold on the Internet to target advertisement.

\section{REFERENCES:}

[1] Malte Burkert, Heiko Krumm, Christoph Fiehe Technical management system for dependable Building Automation Systems 2015 IEEE 20th Conference on Emerging Technologies \& Factory Automation (ETFA). 2015. Pages: 1 - 8, DOI: 10.1109/ETFA.2015.7301656

[2] S. Lasserre, F. Ameziane, D. Haman, A. Fawaz Technical data management systems on large building construction projects. Engineering Management Conference, 2003. IEMC '03. Managing Technologically Driven Organizations: The Human Side of Innovation and Change. 2003. Pages: 110 - 114, DOI: 10.1109/IEMC.2003.1252242

[3] M. G. Ippolito, G. Zizzo, A. Piccolo, P. Siano Definition and application of innovative control logics for residential energy optimization 2014 International Symposium on Power Electronics, Electrical Drives, Automation and Motion. 2014. Pages: 1272 - 1277, DOI: 10.1109/SPEEDAM.2014.6872124

[4] Luigi Martirano A smart lighting control to save energy. Proceedings of the 6th IEEE International Conference on Intelligent Data Acquisition and Advanced Computing Systems. 2011, Volume: 1. Pages: 132 - 138, DOI: 10.1109/IDAACS.2011.6072726

[5] Zhaohui Wu, Jianwei Yin, Shuiguang Deng, Jian Wu, Ying Li, Liang Chen. Modern Service Industry and Crossover Services: Development and Trends in China. IEEE Transactions on Services Computing. 2016, Volume: 9, Issue: 5. Pages: 664 - 671, DOI: 10.1109/TSC.2015.2418765

[6] Usman Raza, Parag Kulkarni, Mahesh Sooriyabandara Low Power Wide Area Networks: An Overview IEEE Communications Surveys \& Tutorials. 2017, Volume: 19, Issue: 2. Pages: 855 - 873, DOI: 10.1109/COMST.2017.2652320 
[7] Keyi Zhang, Alan Marchiori Crowdsourcing low-power wide-area IoT networks 2017 IEEE International Conference on Pervasive Computing and Communications (PerCom). 2017. Pages: 41 - 49, DOI: 10.1109/PERCOM.2017.7917849

[8] AAmir Shahzad, Shahrulniza Musa, Abdulaziz Aborujilah, Muhammad Irfan. Secure Cryptography Testbed Implementation for SCADA Protocols Security. 2013 International Conference on Advanced Computer Science Applications and Technologies. 2013. Pages: 315 320, DOI: 10.1109/ACSAT.2013.69

[9] R. M. J. Rathnayaka, KTMU Hemapala. Developing of scalable SCADA in view of acquiring multi-protocol smart grid devices. 2016 2nd International Conference on Advances in Electrical, Electronics, Information, Communication and Bio-Informatics (AEEICB). 2016. Pages: 182 - 187, DOI: 10.1109/AEEICB.2016.7538269

[10]Jan Markendahl, Osten Makitalo Analysis of Business Models and Market Players for Local Wireless Internet Access 2007 6th Conference on Telecommunication Techno-Economics. 2007. Pages: 1 - 8, DOI: 10.1109/CTTE.2007.4389894 